\title{
Detection of enterovirus D68 in patients hospitalised in three tertiary university hospitals in Germany, 2013 to 2014
}

S Böttcher ${ }^{1}$, C Prifert ${ }^{2}$, B Weißbrich ${ }^{2}, 0$ Adams ${ }^{3}$, S Aldabbagh ${ }^{4}$, AM Eis-Hübinger ${ }^{4}$, S Diedrich ${ }^{1}$

1. National Reference Centre for Poliomyelitis and Enteroviruses, Robert Koch-Institute, Germany

2. Institute of Virology and Immunobiology, University of Würzburg, Germany

3. Institute of Virology, University Hospital of Düsseldorf, Germany

4. Institute of Virology, University of Bonn Medical Centre, Germany

Correspondence: Sabine Diedrich (diedrichs@rki.de)

Citation style for this article:

Böttcher S, Prifert C, Weißbrich B, Adams 0, Aldabbagh S, Eis-Hübinger AM, Diedrich S. Detection of enterovirus D68 in patients hospitalised in three tertiary

university hospitals in Germany, 2013 to 2014. Euro Surveill. 2016;21(19):pii=30227. DOI: http://dx.doi.org/10.2807/1560-7917.ES.2016.21.19.30227

Enterovirus D68 (EV-D68) has been recognised as a worldwide emerging pathogen associated with severe respiratory symptoms since 2009. We here report EV-D68 detection in hospitalised patients with acute respiratory infection admitted to three tertiary hospitals in Germany between January 2013 and December 2014. From a total of 14,838 respiratory samples obtained during the study period, 246 (1.7\%) tested enterovirus-positive and, among these, 39 (15.9\%) were identified as EV-D68. Infection was observed in children and teenagers $(0-19$ years; $n=31)$, the majority $(n=22)$ being under five years-old, as well as in adults > 50 years of age $(n=8)$. No significant difference in prevalence was observed between the 2013 and 2014 seasons. Phylogenetic analyses based on viral protein 1 (VP1) sequences showed co-circulation of different EV-D68 lineages in Germany. Sequence data encompassing the entire capsid region of the genome were analysed to gain information on amino acid changes possibly relevant for immunogenicity and revealed mutations in two recently described pleconaril binding sites.

\section{Introduction}

Within the picornaviridae family the genus Enterovirus is known to include more than 120 human enterovirus (EV) serotypes, causing a broad range of symptoms mainly in children below the age of five years. The major clinically relevant manifestations of non-polio enteroviruses (NPEV) include meningitis/encephalitis or acute flaccid paralyses (AFP), atypical hand, foot and mouth-disease or myocarditis. Some serotypes have been identified to be predominantly associated with respiratory diseases. Of those, EV-D68 has, since its first description in 1962, been detected sporadically worldwide until 2009 [1]. Subsequently, several epidemic clusters of EV-D68 associated with increases of respiratory illnesses have been reported, $[1,2]$. The largest outbreak so far was reported in autumn 2014 from the United States (US) with more than 1,100 EV-D68 detections in children hospitalised with acute severe respiratory infections $[1,3]$.

In Germany, surveillance of respiratory virus infections is conducted mainly with regards to influenza representing a vaccine preventable disease, and is based on sentinel surveillance systems including outpatients with influenza like illness (ILI) and/or acute respiratory infection (ARI) (AGI Influenza RKI [4]; ARE NLGA [5]). Furthermore, a laboratory network reporting detection of respiratory viruses in hospitalised patients was established in 2009 (RespVir [6]). Besides influenza, pathogens recorded within these systems include respiratory syncytial virus (RSV), human metapneumovirus (HMPV), parainfluenza viruses (HPIV), coronaviruses ( $\mathrm{HCoV}$ ), adenoviruses (HAdV), rhinoviruses (HRV), and EV. Since the latter viruses are not routinely differentiated, no valid data on EV circulation including EV-D68 in Germany are available.

The aim of the study was to investigate the prevalence of EV-D68 in Germany by analysing EV-positive respiratory tract samples collected from patients admitted to three German university hospitals in two consecutive years. Furthermore, nucleotide (nt) sequence analysis of the complete viral protein $1\left(\mathrm{VP}_{1}\right)$ region was performed for comparison of EV-D68 strains circulating in Germany with recent published strains from other countries. Complete capsid sequences from selected strains based on phylogenetic analysis were obtained to provide more data for better understanding of any changes in antigenicity. 
Number of enterovirus (EV)-positive samples obtained by three university laboratoriesa stratified as EV-D68 and non-

EV-D68, by week, Germany, 2013-2014 (n=246)

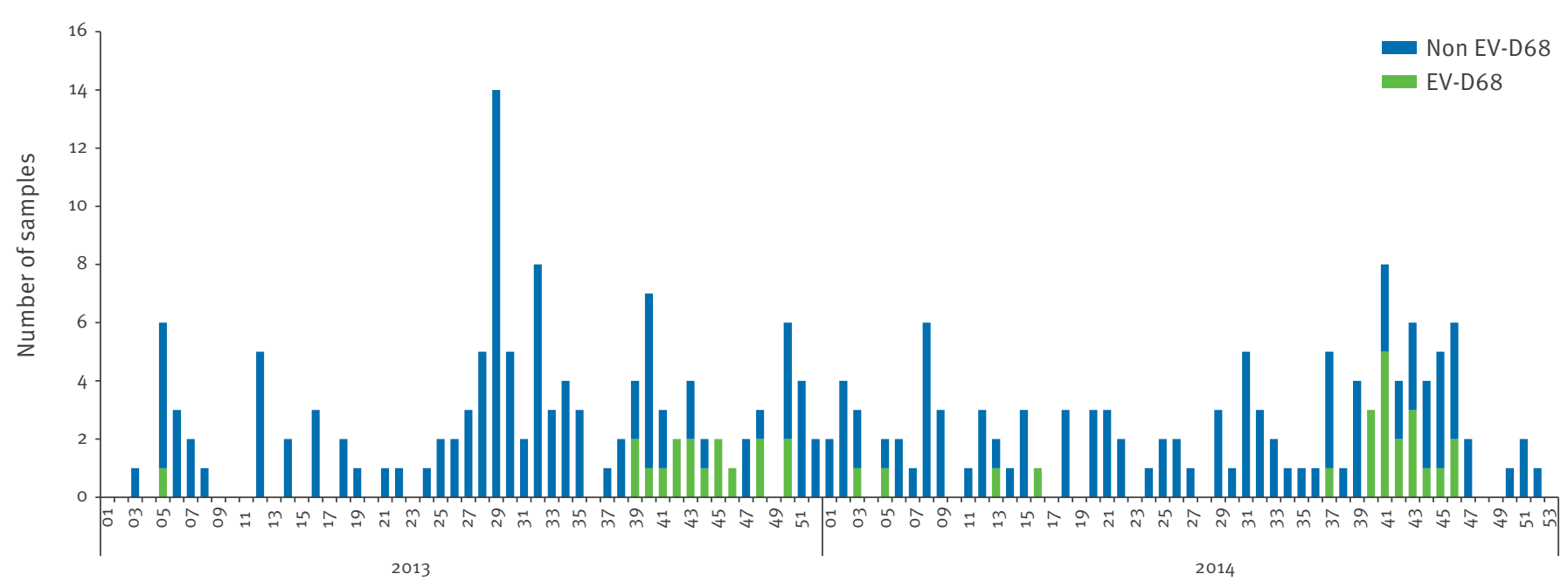

Calendar weeks $2013-2014$

a The laboratories were the Institute of Virology and Immunobiology, University of Würzburg, the Institute of Virology, University Hospital of Düsseldorf and the Institute of Virology, University of Bonn Medical Centre.

\section{Methods}

\section{Setting}

Three German university laboratories provided data and samples collected from January 2013 through December 2014 to this study: the Institute of Virology and Immunobiology, University of Würzburg (laboratory 1), the Institute of Virology, University Hospital of Düsseldorf (laboratory 2) and the Institute of Virology, University of Bonn Medical Centre (laboratory 3).

\section{Sample collection}

Respiratory samples (e.g. nasopharyngeal swabs, bronchial lavages) were collected from patients with respiratory diseases admitted to the affiliated tertiary hospitals. The samples were routinely screened for a broad panel of respiratory pathogens including EV/HRV and other respiratory viruses (influenza $A$ and $B, R S V$, HMPV, HPIV 1-4, HCoV 229, NL63, HKU1, OC43, HAdV, parechoviruses, bocavirus) according to the individual laboratory protocols. All samples positive for EV or EV/ HRV were included in this study. These EV samples represent about one fourth of the overall number of EV positive samples detected in the nationwide RespVir surveillance [6].

The diagnostic procedures for the detection of respiratory viruses of the three university laboratories are as follows: laboratory 1: FTD 'Respiratory Pathogens 21' (Fast track Diagnostics, Luxembourg), laboratory 2: Bonzel et al., 2008 [7], laboratory 3: Dierssen et al., 2008 [8] and Poelman et al., 2014 [9]. All methods have been proven to detect EV-D68 in national and international proficiency tests.
Polymerase chain reaction amplification of enterovirus D68 viral protein 1 region

For highly sensitive amplification of the complete VP1 region of EV-D68 strains directly from clinical material a specific one-step reverse-transcription polymerase chain reaction (RT-PCR) assay was established at the German National Reference Centre for Poliomyelitis and Enteroviruses (NRZ PE). Amplification was performed using One-Step-RT-PCR Kit (Qiagen, Hilden, Germany) followed by a nested PCR using HotStarTaqMastermix (Qiagen, Hilden Germany) according to the manufacturer's protocol. RT-PCR and nested PCR were done with $600 \mathrm{nM}$ of primers (Table 1). The RT-PCR was conducted with primers NRZ $267 / 268$ and with the following temperature profile: $10 \min 22^{\circ} \mathrm{C}, 45 \min 50^{\circ} \mathrm{C}$, $15 \mathrm{~min} 95^{\circ} \mathrm{C}$ for RT followed by 40 cycles $\left(30 \mathrm{~S} 94^{\circ} \mathrm{C}\right.$; $30 \mathrm{~S} 55^{\circ} \mathrm{C} ; 90 \mathrm{~S} 72^{\circ} \mathrm{C}$ ) and final elongation for $10 \mathrm{~min}$ at $72^{\circ} \mathrm{C}$. The nested PCR was carried out with primers $269 / 270$ by using a touchdown protocol with 10 cycles (30 S $94^{\circ} \mathrm{C} ; 30 \mathrm{~s} 60^{\circ} \mathrm{C} ; 90 \mathrm{~S} 72^{\circ} \mathrm{C}$ ) with a decrease of $1^{\circ} \mathrm{C}$ per cycle of the initial $60^{\circ} \mathrm{C}$ annealing temperature, followed by 30 cycles $\left(30594^{\circ} \mathrm{C}\right.$; $30550^{\circ} \mathrm{C}$; $90 \mathrm{~s} 72^{\circ} \mathrm{C}$ ) and final elongation for $10 \mathrm{~min}$ at $72^{\circ} \mathrm{C}$. The resulting product of 1,129 bp was treated with ExoSAP-IT (Affymetrix) before cycle sequencing with primers NRZ 269, NRZ 270 and NRZ 271 using the BigDye 3.1 kit (Applied Biosystems, Weiterstadt, Germany).

\section{Phylogenetic analysis}

Sequences were assembled using Sequencher software version 5.2.4. Alignments were performed using MAFFT [10] and the phylogenetic relationships among the strains circulating in Germany and representative strains taken from GenBank were estimated using 


\section{FIGURE 2}

Phylogenetic analysis ${ }^{\mathrm{a}}$ of enterovirus D68 sequences $(\mathrm{n}=37)$ obtained by three university laboratories ${ }^{\mathrm{b}}$, Germany, 2013-2014

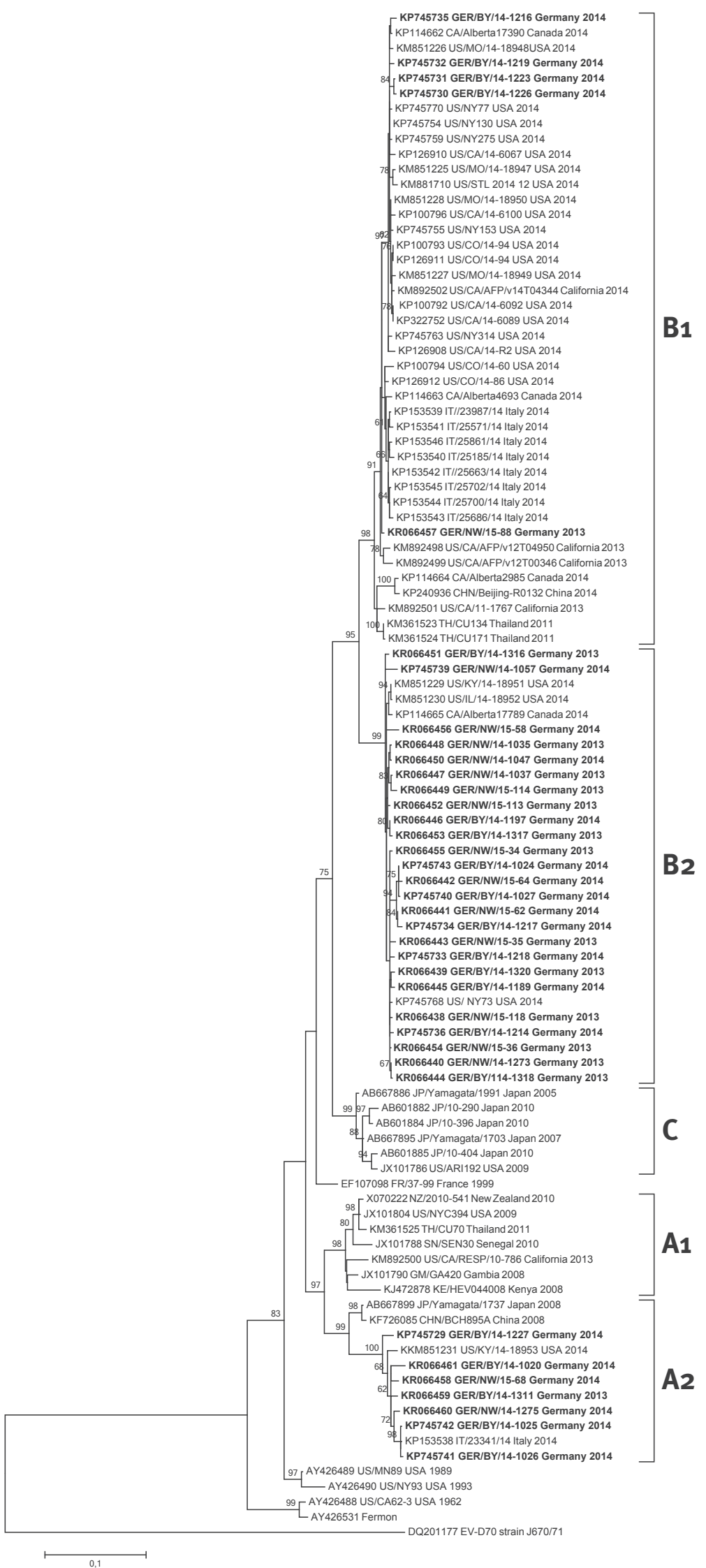

The phylogenetic analysis is based on the complete viral protein 1 region. Bootstrap values are shown at the nodes. The scale bar indicates the number of nucleotide (nt) substitutions per site.

${ }^{a}$ The complete VP1 region nt sequence corresponded to bases 2,389-3,315 of EV-D68 prototype strain Fermon (GenBank accession number: AY426531).

${ }^{b}$ The laboratories were the Institute of Virology and Immunobiology, University of Würzburg, the Institute of Virology, University Hospital of Düsseldorf and the Institute of Virology, University of Bonn Medical Centre. 
the maximum likelihood (ML) method based on the Tamura-Nei model conducted with molecular evolutionary genetics analysis (MEGA6) using a bootstrap procedure with 1,000 replicates [11].

\section{Molecular typing of non-enterovirus D68 enteroviruses}

Molecular typing of non-EV-D68 enteroviruses was carried out by sequencing of the $V P_{1}$ region using published PCR systems with slightly modified conditions due to use of the Qiagen One Step RT-PCR kit instead of Invitrogen Superscript II and III as described in references $[12,13]$. Details of methodology are available upon request. For those samples remaining VP1 PCR negative, sequencing of partial 5'non-coding region (5'NCR) [14] allowed assignment to enterovirus group A-D. Samples with no clear basic local alignment search tool (BLAST) result were classified as NPEV.

\section{Analysis of immunogenic sites in the capsid} proteins of enterovirus D68

To provide sequence data for further understanding of possible changes in the immunogenic sites of the capsid, 23 strains representing members of all three current subclades (A2, B1, B2) were selected for sequencing of the entire capsid $\left(\mathrm{P}_{1}\right)$ genomic region encoding all four capsid proteins as well as adjacent $5^{\prime} \mathrm{NCR}$ region. Amplification of the $\mathrm{VP}_{4} / \mathrm{VP}_{2} / \mathrm{VP}_{3}$ region of the genome from clinical material was performed with primers listed in Table 1 using the following cycling protocol: $45 \min 50^{\circ} \mathrm{C}, 15 \mathrm{~min} 95^{\circ} \mathrm{C}$ followed by 25 cycles ( $30 \mathrm{~S} 94^{\circ} \mathrm{C} ; 30 \mathrm{~S} 55^{\circ} \mathrm{C} ; 90 \mathrm{~S} 72^{\circ} \mathrm{C}$ ) and final elongation for $10 \mathrm{~min}$ at $72^{\circ} \mathrm{C}$. Nested PCR was carried out $15 \mathrm{~min}$ at $95^{\circ} \mathrm{C}$ followed by 25 cycles $\left(30 \mathrm{~S} 94^{\circ} \mathrm{C}\right.$; $30 \mathrm{~S} 55^{\circ} \mathrm{C} ; 30 \mathrm{~S} 72^{\circ} \mathrm{C}$ ) and final elongation for $10 \mathrm{~min}$ at $72^{\circ} \mathrm{C}$. PCR products were directly sequenced after EXOSAP-IT treatment with primers used for nested PCR. Amplification and sequencing of partial $5{ }^{\prime} \mathrm{NCR}$ was done as described recently [14].

\section{Results}

\section{Enterovirus D68 detection}

From January 2013 to the end of December 2014, 14,838 respiratory samples from patients admitted to three tertiary university hospitals were analysed, with 246 $(1.7 \%)$ being EV- positive. EV-positive samples were retrospectively typed with molecular methods resulting in a total of $39 \mathrm{EV}$-D68 detections with 17 (0.2\%) detections in 2013 and 22 (0.3\%) detections in 2014 (Table 2).

When analysed in more detail, variations in EV-D68 prevalence among patients admitted to each of the three hospitals were noticed. While in one hospital a moderate raise in EV-D68 infections among total samples analysed was observed in 2014 compared with 2013 (0.2\% in 2013 vs $0.6 \%$ in 2014), another hospital showed a higher EV-D68 rate in 2013 (0.6\% in 2013 vs $0.1 \%$ in 2014).
Weekly distribution of EV-D68 positive samples, as shown in Figure 1, peaked in late summer and autumn months (September-November). This was also reflected by the EV-D68-positivity rates among EV-positive samples, which in calendar weeks 36 to 48 corresponding to September to November (last column, Table 2) ranged from 23.8 to $54.5 \%$. Regarding the individual hospitals, EV-D68 was detected nearly consistently among EVs during weeks 36 to 48 in hospital 1 and 2 (54.5\% in 2013 vs $50.0 \%$ in 2014; and $27.3 \%$ in 2013 vs $23.8 \%$ in 2014). Hospital 3 showed higher EV-D68 rate in 2013 (45.5\%) than in 2014 (25.0\%), however, some caution is needed concerning this hospital because of the relatively small number of EV-D68- positive samples which might be biased by the overall low number of EV detections. On average, no substantial differences in EV-D68 rates could be found between 2013 and 2014 suggesting two regular seasons.

The 39 EV-D68 samples detected over the whole study period were from young children aged $0-9$ years $(n=28)$, teenagers aged $10-19$ years $(n=3)$ and adults aged $>50$ years $(n=8)$. Within the group of young children, the majority of EV-D68 patients was under the age of five years $(n=22)$. The male/female ratio for EV-D68-positive patients was 1.4:1 $(m=23, f=16)$. Co-infection with other viruses was observed in two EV-D68-positive samples (Cox A10, $n=1 ; \mathrm{HCoV} \mathrm{OC}_{43}$, $n=1$ ). Specified clinical details were not accessible for all EV-D68 patients. For patients where data were available $(n=15)$, pneumonia $(n=6)$ or obstructive bronchitis $(n=6)$ were most commonly reported.

\section{Amplification and phylogenetic analysis based} on viral protein 1 region

Amplification of the complete VP1 sequence was achieved for 37 of 39 EV-D68 samples. For phylogenetic analysis complete VP1 sequences of EV-D68 strains available in GenBank were used. The ML tree confirmed the previously observed divergence of EV-D68 strains circulating since 2005 into three major subgroups A, B, and C (Figure 2) [15]. Furthermore, as recently described, subgroups $A$ and $B$ segregated in two subclades [16]. Among the 37 EV-D68 strains, seven belonged to clade $A 2$ and 30 belonged to clade $B$ $\left(B_{1}: n=5, B 2: n=25\right)$. All sequences were deposited in GenBank under accession numbers KP745729-43 and KRo66438-61.

Amplification and sequencing of partial $\mathrm{VP}_{1}$ region from non-EV-D68 viruses revealed serotypes from enterovirus species EV-A $(n=87)$ and EV-B $(n=68)$. Typing results are shown in more detail in Table 3. Samples remaining negative in $V P_{1}$ amplification were categorised as EV-A $(n=3)$, EV-B $(n=6)$, and EV-C $(n=1)$ by $5^{\prime}$ NCR sequencing. Fifteen samples were identified as rhinovirus (HRV-A: $n=8$, HRV-C: $n=7$ ). Twenty-nine samples gave no clear BLAST result and were classified as NPEV (Table 3). 


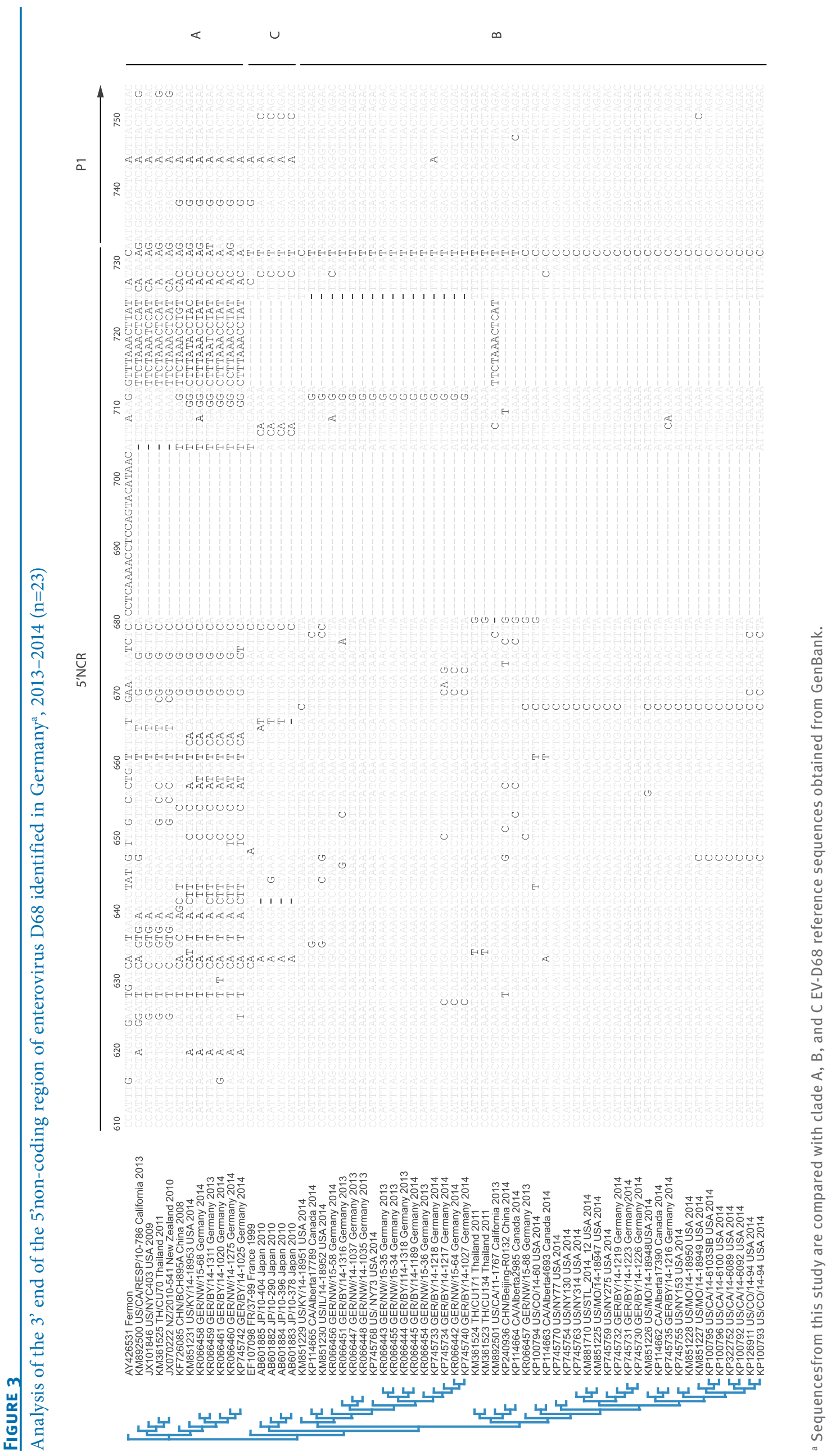

www.eurosurveillance.org

5 
TABLE 1

Primers used for amplification and sequencing of the complete capsid (P1) region of enterovirus D68

\begin{tabular}{|c|c|c|c|}
\hline Target region and primer & Sequence $5^{\prime}-3^{\prime}$ & Orientation & Location $^{\mathrm{a}}$ \\
\hline \multicolumn{4}{|l|}{$\mathrm{VP}_{1}$} \\
\hline NRZ 267 & ATG YTA GST ACW CAT RTB GTB TGG GAY TT & Sense & $2,125-2,153$ \\
\hline NRZ 268 & ATC CAY TGR ATM CCW GGG CCY TCR AAR C & Antisense & $3,557-3,530$ \\
\hline NRZ 269 & AAT GCY AAY GTT GGY TAY GTY ACH TGT T & Sense & $2,239-2,266$ \\
\hline NRZ 270 & AAG AYC CYA CAA ARA CYC CHC CRW ARC CKG G & Antisense & $3,358-3,327$ \\
\hline NRZ 271 & CAA GCA ATG TTY GTA CCH ACT GG & Sense & $2,854-2,876$ \\
\hline \multicolumn{4}{|l|}{$\mathrm{VP}_{2} / 4$} \\
\hline NRZ 272 & GTG GTC CAG GCT GCG TTG GCG & Sense & $350-370$ \\
\hline NRZ 273 & TTR AAC TCA CAA CAC ATT GGA GCR ATT G & Antisense & $1,658-1,631$ \\
\hline NRZ 274 & ATG AAC AAG GTG TGA AGA GTC TAT TGA GC & Sense & $405-433$ \\
\hline NRZ 275 & ACT GGT ATT ATT GCT AGY GTC CAC TG & Antisense & $1,580-1,555$ \\
\hline \multicolumn{4}{|l|}{$\mathrm{VP}_{3}$} \\
\hline NRZ 276 & TGA CAT CAT GAA AGG TGA AGA AGG AGG & Sense & $1,371-1,397$ \\
\hline NRZ 277 & GTG CGA GTT TGT ATG GCT TCY TCT GG & Antisense & $2,564-2,539$ \\
\hline NRZ 278 & GTT CTT CCC TGG ATG AAT GCY GCT CC & Sense & $1,504-1,529$ \\
\hline NRZ 279 & CTC TCR ATY TGR TAG GCT GCC TCT G & Antisense & $2,432-2,408$ \\
\hline
\end{tabular}

VP: viral protein.

a Nucleotide locations are relative to the genome of EV-D68 prototype strain Fermon (GenBank accession number: AY426531).

Analysis of complete capsid region (P1) amino acid- and partial 5'non-coding region sequences

Twenty-three EV-D68 sequences of strains belonging to subclades A2, B1, and B2 from this study were compared with 40 sequences available through GenBank. There were only few amino acids exclusively defining a single clade, however clade $A$ was characterised by $E_{143}$ and $V_{2} 91$ in VP2, N525 and $V_{53} 3$ in VP3 and a deletion of N692 in VP1. Strains assigned to subclade $A_{2}$ carried an arginine and lysine insertion at position 859 of VP1. No differences between strains circulating in 2014 and strains circulating before 2014 were observed with regard to the defined loop structures of the capsid proteins $V P_{1}, V P_{2}$, and $V_{3}$ representing neutralising immunogenic sites ( $\mathrm{VP} 2 \mathrm{EF}$ loop, $\mathrm{VP}_{3} \mathrm{BC}$ loop, $\mathrm{VP}_{1} \mathrm{BC}$ loop and DE loop; alignment available upon request). Notably, two amino acid positions that have been reported to interact with the antiviral pleconaril differed in strains assigned to subclade B1 compared with

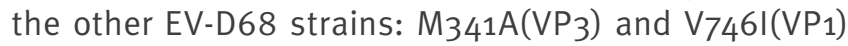
[17]. Whether or not these changes influence pleconaril efficacy requires experimental confirmation.

Within the $3^{\prime}$ end of the $5^{\prime} \mathrm{N} C R$, all strains included in the comparison showed a 23 or 24 nt deletion (681703/704 compared with prototype strain Fermon). In addition, all B and C strains carried a 12 or 13 nt deletion (713-724/725 compared with Fermon), except strain KM892501 (Figure 3).

\section{Discussion}

In this study we provide epidemiological and phylogenetic information on EV-D68 in hospitalised patients admitted with respiratory diseases to three tertiary hospitals in Germany from January 2013 through December 2014. During this period, EV-D68 circulation appeared to have a seasonal pattern, with an increase in numbers of patient samples testing positive for this virus from the beginning of the autumn until the early winter months. The apparent seasonality was also reflected in the EV-D68 positivity rates among enterovirus-positive samples from September to November (calendar week 36-48), which ranged between $23.8 \%$ and $54.5 \%$, compared to between $8.3 \%$ and $23.4 \%$ annually.

Overall EV-D68 infections could be detected in children and teenagers, with most detections in those under five years-old. Adults over 50 years of age were also affected. The male/female ratio of 1.4:1 among all respiratory isolates indicated a male predominance, which has also been previously described for enterovirus-infected patients [18].

Among the total annual numbers of analysed respiratory samples, EV-D68 was detected at a rate of $0.2 \%$ (2013) and $0.3 \%$ (2014). The similar rates between the two years suggest that each year was characterised by a regular season. In support of this, similar prevalences have been reported for hospitalised patients [19-21] as well as outpatients $[18,22]$ from several studies worldwide in non-epidemic years. In contrast, for years with described increased EV-D68 activity, an overall annual EV-D68 detection rate of $>1 \%$ has been observed in hospitalised patients $[20,23,24]$ as well as outpatients $[22,25]$. 
Overview of respiratory samples analysed and enterovirus (EV) and EV-D68 detection rates by three university laboratories ${ }^{\mathrm{a}}$, Germany, 2013-2014 ( $\mathrm{n}=14,838$ respiratory samples)

\begin{tabular}{|c|c|c|c|c|c|c|}
\hline Laboratory/hospital & Year & $\begin{array}{c}\text { Respiratory } \\
\text { samples } \\
\text { N }\end{array}$ & $\begin{array}{l}\text { EV positive } \\
\mathrm{N} \text { (\% of } \\
\text { respiratory } \\
\text { samples) }\end{array}$ & $\begin{array}{c}\text { EV-D68 positive } \\
\text { N (\% of respiratory } \\
\text { samples) }\end{array}$ & $\begin{array}{l}\text { EV-D68/EV } \\
\text { positives (\%) } \\
\text { annually }\end{array}$ & $\begin{array}{l}\text { Number of EV-D68/EV } \\
\text { positives (\%) in } \\
\text { calendar week } 36-48\end{array}$ \\
\hline \multirow{2}{*}{1} & 2013 & 3,526 & $46(1.3)$ & $6(0.2)$ & $6 / 46(13.0)$ & $6 / 11(54.5)$ \\
\hline & 2014 & 2,696 & $64(2.4)$ & $15(0.6)$ & $15 / 64(23.4)$ & $12 / 24(50.0)$ \\
\hline \multirow{2}{*}{2} & 2013 & 3,351 & $55(1.6)$ & $6(0.2)$ & $6 / 55(10.9)$ & $3 / 11(27.3)$ \\
\hline & 2014 & 3,753 & $44(1.2)$ & $6(0.2)$ & $6 / 44(13.6)$ & $5 / 21(23.8)$ \\
\hline \multirow[b]{2}{*}{3} & 2013 & 813 & $25(3.1)$ & $5(0.6)$ & $5 / 25(20)$ & $5 / 11(45 \cdot 5)$ \\
\hline & 2014 & 699 & $12(1.7)$ & $1(0.1)$ & $1 / 12(8.3)$ & $1 / 4(25.0)$ \\
\hline \multicolumn{2}{|l|}{ Total 2013} & 7,690 & $126(1.6)$ & $17(0.2)$ & $17 / 126(13.5)$ & $14 / 33(42.4)$ \\
\hline \multicolumn{2}{|l|}{ Total 2014} & 7,148 & $120(1.7)$ & $22(0.3)$ & $22 / 120(18.3)$ & $18 / 49(36.7)$ \\
\hline \multicolumn{2}{|l|}{ Total 2013-2014 } & 14,838 & 246 (1.7) & $39(0.3)$ & $39 / 246(15.8)$ & $32 / 82(39.0)$ \\
\hline
\end{tabular}

a The laboratories were the Institute of Virology and Immunobiology, University of Würzburg, the Institute of Virology, University Hospital of Düsseldorf and the Institute of Virology, University of Bonn Medical Centre.

A more detailed data analysis revealed variation in EV-D68 prevalence among patients admitted to each of the three hospitals in our study between the years, suggesting a broad range in EV-D68 positivity rates from one season to another. The annual rates of EV-D68 positivity among all respiratory samples for each hospital during the study period remained however $\ll 1 \%$.

Worldwide reports on the detection of EV-D68 in patients with respiratory diseases increased rapidly during the last few years especially during the 2008 to 2010 period [2]. The observation of an upsurge in hospitalised patients due to EV-D68 infection in the US and Canada in $2014[1,3,26]$ resulted in the recognition of EV-D68 as an (re)emerging pathogen. In response to this, the European Society for Clinical Virology (ESCV) launched a study in collaboration with the European Centre for Disease Prevention and Control (ECDC) to collect information on EV-D68 infections in paediatric patients in September/October 2014 in Europe [16]. We contributed to this study and therefore samples collected between September 2014 and November 2014 reported here were also included in that study. However, no epidemiological data on EV-D68 in Germany covering a period as extended as this current study have been described so far. The only data available to date came from an EV-D68-specific screening of samples collected within the German ILI/ARI outpatient study during the August to October 2014 period, resulting in identification of 25 EV-D68-positive samples among 325 samples (7.7\%) screened [27].

Coinciding with the upsurge of severe acute respiratory diseases in the US and Canada in 2014, a cluster of 12 paediatric patients with AFP following respiratory illness was reported from Colorado [28,29]. Among 11 of these children, five ( $45 \%)$ tested EV-D68 positive in respiratory specimens $[28,29]$. A further investigation of AFP cases reported nationwide in the US during the same period (August through October) found 88 cases of AFP in 32 States, revealing a similar EV-D68 positive rate [30]. In contrast, no development of central nervous system (CNS) complications was reported in the patients from this study. Furthermore, in the context of the Global Polio Eradication Initiative programme, the German enterovirus surveillance [31] reported no significant increase of AFP cases in 2013 and 2014 compared to the 2006 to 2014 average in Germany (Katrin Neubauer, personal communication 15 May 2015).

As part of the EVSurv, laboratory diagnostics focus mainly on stool samples from patients with symptoms of aseptic meningitis/encephalitis and/or AFP to exclude polioviruses. Stool samples are nevertheless not suitable for EV-D68 detection, due to the biological properties of this virus $[32,33]$. In spite of this, among 24,246 specimens tested for enteroviruses between 2006 and 2014 within the EVSurv, three stool samples from paediatric patients with signs of aseptic meningitis were reported as EV-D68-positive (2 in 2010, 1 in 2013). These most probably resulted from spill-over from the respiratory tract, but nevertheless suggest circulation of EV-D68 in Germany before 2013 and presumably possible association with CNS disorders.

As different EV-D68 clades are evolving over time [15], the increased detection of EV-D68 in the recent decade may have been due to changes in antigenicity [34]. Comparison of the complete capsid sequences of 23 strains isolated in 2013 and 2014 in Germany with reference strains obtained from GenBank did not reveal any amino acid residues in antigenic sites that were unique to the 2013 or 2014 strains.

Furthermore, all German strains described here showed clade specific deletions within the 3' end of 5'NCR as reviewed in Imamura and Oshitani in 2014 [2]. All clade A strains identified in this study displayed 


\section{TABLE 3}

Enterovirus typing results from respiratory samples, Germany, 2013-2014 ( $\mathrm{n}=207$ samples) ${ }^{\mathrm{a}}$

\begin{tabular}{|c|c|c|}
\hline Species & Serotype & Number ${ }^{a}$ \\
\hline \multirow{9}{*}{ EV-A } & Cox A10 & 12 \\
\hline & $\mathrm{Cox} \mathrm{A}_{2}$ & 8 \\
\hline & $\mathrm{Cox} \mathrm{A}_{4}$ & 21 \\
\hline & $\mathrm{Cox} \mathrm{A}_{5}$ & 3 \\
\hline & Cox A6 & 23 \\
\hline & Cox A8 & 1 \\
\hline & Cox A16 & 10 \\
\hline & EV-A71 & 9 \\
\hline & NA & 3 \\
\hline \multirow{13}{*}{ EV-B } & $\mathrm{Cox} B 2$ & 6 \\
\hline & $\mathrm{Cox} \mathrm{B}_{3}$ & 8 \\
\hline & $\mathrm{Cox} \mathrm{B}_{4}$ & 7 \\
\hline & $\mathrm{Cox} \mathrm{B}_{5}$ & 5 \\
\hline & Echo 11 & 2 \\
\hline & Echo 18 & 5 \\
\hline & Echo 25 & 2 \\
\hline & Echo 27 & 1 \\
\hline & Echo 3 & 5 \\
\hline & Echo 6 & 1 \\
\hline & Echo 30 & 19 \\
\hline & $\mathrm{Cox} \mathrm{A}_{9}$ & 7 \\
\hline & NA & 6 \\
\hline EV-C & NA & 1 \\
\hline EV-D & EV-D68 & 39 \\
\hline HRV A & $N A$ & 8 \\
\hline HRV C & NA & 7 \\
\hline NPEV & NA & 29 \\
\hline
\end{tabular}

NA: not applicable.

a Due to two samples carrying two different serotypes, the total number of serotypes in the table differ from the total number of samples analysed.

the previously described deletion within the DE loop in VP1 [21]. Interestingly, all German strains assigned to subclade $A_{2}$ were identified in seven of eight adult patients. Those strains carried a subclade A2 specific insertion of two amino acids (arginine and lysine) at the very end of the C-terminus of VP1. So far, no function has been assigned to this region. Comparison with prototype sequences of all other known enterovirus serotypes revealed a highly diverse C-terminus of VP1 downstream from a conserved proline-rich region (data not shown, available upon request). Furthermore, we recognised two amino acid exchanges in subclade $B_{1}$ strains ( $\mathrm{M}_{341 \mathrm{~A}}$ in $\mathrm{VP}_{3}$ and $\mathrm{V}_{746}$ in $\mathrm{VP}_{1}$ ) at positions that have been described to interact with pleconaril [17] (data not shown, available upon request).

In summary, no significant changes in the EV-D68 prevalence in patients admitted to three German tertiary hospitals could be observed in 2014 compared with 2013. On the basis of amino acid sequences of the capsid proteins no unique changes in 2014 strains compared with 2013 strains or clade specific differences were found. However, the insertion of two amino acids at the C-terminus of VP1 of subclade $\mathrm{A}_{2}$ strains, combined with their occurrence in adults, warrants further experimental investigation regarding neutralisation properties of antibodies directed against (i) the strains not containing this insertion versus (ii) the strains containing the insertion. Furthermore, continuous molecular surveillance of enteroviruses in respiratory samples using defined criteria is a necessity to be able to interpret potential epidemiological and clinical situations like those recently reported from North America and Canada.

Acknowledgements

We thank the technical staff in all four labs for excellent performance.

\section{Conflict of interest}

None declared.

\section{Authors' contributions}

Sindy Böttcher has drafted the manuscript, initiated the study and developed and performed EV-D68 specific PCRassays. Christiane Prifert performed EV/HRV screening and conducted phylogenetic analyses. Benedikt Weißbrich provided respiratory samples for enterovirus characterisation. Ortwin Adams provided respiratory samples for enterovirus characterisation. Souhaib Aldabbagh performed EV/HRV screening. Anna-Maria Eis-Hübinger provided respiratory samples for enterovirus characterization. Sabine Diedrich conceptualised the study and has participated in writing of the manuscript together with Sindy Böttcher.

References

1. European Centre for Disease Prevention and Control (ECDC). Enterovirus 68 rapid risk assessment updated. Stockholm: ECDC 24; Nov. 2014. Available from: http://www.ecdc. europa.eu/en/press/news/_layouts/forms/News_DispForm. aspx?List=8db7286c-fe2d-476c-9133-18ff4cb1b568\&ID=1125

2. Imamura T, Oshitani H. Global reemergence of enterovirus D68 as an important pathogen for acute respiratory infections. Rev Med Virol. 2015;25(2):102-14. DOI: 10.1002/rmv.1820 PMID: 25471236

3. Midgley CM, Jackson MA, Selvarangan R, Turabelidze G, Obringer $E$, Johnson $D$, et al. Severe respiratory illness associated with enterovirus D68 - Missouri and Illinois, 2014. MMWR Morb Mortal Wkly Rep. 2014;63(36):798-9.PMID: 25211545

4. der Heiden MA, Köpke K, Buda S, Buchholz U, Haas W. Estimates of excess medically attended acute respiratory infections in periods of seasonal and pandemic influenza in Germany from 2001/02 to 2010/11.PLoS One. 2013;8(7):e64593. DOI: 10.1371/journal.pone.0064593 PMID: 23874380

5. Niedersächsische Landesgesundheitsamt (NLGA). Akute respiratorische Erkrankungen (ARE). NLGA. German. [Accessed 20 May 2015]. Available from: www.nlga.niedersachsen.de

6. Respiratorisches Viren - Netzwerk (RespVir). German. [Accessed 20 May 2015]. Available from: http://rvdev.medicaldpc.com/

7. Bonzel L, Tenenbaum T, Schroten H, Schildgen O, SchweitzerKrantz S, Adams O. Frequent detection of viral coinfection in children hospitalized with acute respiratory tract infection using a real-time polymerase chain reaction. Pediatr Infect Dis J. 2008;27(7):589-94. DOI: 10.1097/INF.ob013e3181694fb9 PMID: 18520973 
8. Dierssen U, Rehren F, Henke-Gendo C, Harste G, Heim A. Rapid routine detection of enterovirus RNA in cerebrospinal fluid by a one-step real-time RT-PCR assay.J Clin Virol. 2008;42(1):58-64. DOI: 10.1016/j.jcv.2007.11.016 PMID: 18164234

9. Poelman R, Schölvinck EH, Borger R, Niesters HG, van Leer-Buter C. The emergence of enterovirus D68 in a Dutch University Medical Center and the necessity for routinely screening for respiratory viruses.J Clin Virol. 2015;62:1-5. DOI: 10.1016/j.jcv.2014.11.011 PMID: 25542461

10. Katoh K, Standley DM. MAFFT multiple sequence alignment software version 7: improvements in performance and usability.Mol Biol Evol. 2013;30(4):772-80. DOI: 10.1093/ molbev/msto10 PMID: 23329690

11. Tamura K, Stecher G, Peterson D, Filipski A, Kumar S. MEGA6: Molecular Evolutionary Genetics Analysis version 6.0.Mol Bio Evol. 2013;30(12):2725-9. DOI: 10.1093/molbev/mst197 PMID: 24132122

12. Nix WA, Oberste MS, Pallansch MA. Sensitive, seminested PCR amplification of VP1 sequences for direct identification of all enterovirus serotypes from original clinical specimens.J Clin Microbiol. 2006;44(8):2698-704. DOI: 10.1128/JCM.00542-06 PMID: 16891480

13. Leitch EC, Harvala H, Robertson I, Ubillos I, Templeton K, Simmonds P. Direct identification of human enterovirus serotypes in cerebrospinal fluid by amplification and sequencing of the VP1 region.J Clin Virol. 2009;44(2):119-24. DOI: 10.1016/j.jcv.2008.11.015 PMID: 19135410

14. Kuryk $t$, Wieczorek M, Diedrich S, Böttcher S, Witek A, Litwińska B. Genetic analysis of poliovirus strains isolated from sewage in Poland.J Med Virol. 2014;86(7):1243-8. DOI: 10.1002/jmv.23803 PMID: 24123142

15. Tokarz R, Firth C, Madhi SA, Howie SR, Wu W, Sall AA, et al. Worldwide emergence of multiple clades of enterovirus 68 . Gen Virol. 2012;93(Pt 9):1952-8. DOI: 10.1099/vir.0.043935-0 PMID: 22694903

16. Poelman R, Schuffenecker I, Van Leer-Buter C, Josset L, Niesters HG, Lina B, ESCV-ECDC EV-D68 study group. European surveillance for enterovirus D68 during the emerging NorthAmerican outbreak in 2014.J Clin Virol. 2015;71:1-9. DOI: 10.1016/j.jcv.2015.07.296 PMID: 26364237

17. Liu Y, Sheng J, Fokine A, Meng G, Shin WH, Long F, et al. Structure and inhibition of EV-D68, a virus that causes respiratory illness in children. Science. 2015;347(6217):71-4 DOI: $10.1126 /$ science.1261962 PMID: 25554786

18. Meijer A, Benschop KS, Donker GA, van der Avoort HG. Continued seasonal circulation of enterovirus D68 in the Netherlands, 2011-2014.Euro Surveill. 2014;19(42):20935. DOI: 10.2807/1560-7917.ES2014.19.42.20935 PMID: 25358039

19. Piralla A, Girello A, Grignani M, Gozalo-Margüello M, Marchi A, Marseglia G, et al. Phylogenetic characterization of enterovirus 68 strains in patients with respiratory syndromes in Italy. J Med Virol. 2014;86(9):1590-3. DOI: 10.1002/ jmv.23821 PMID: 24155220

20. Ikeda T, Mizuta K, Abiko C, Aoki Y, Itagaki T, Katsushima F, et al. Acute respiratory infections due to enterovirus 68 in Yamagata, Japan between 2005 and 2010. Microbiol Immunol. 2012;56(2):139-43. DOI: 10.1111/j.1348-0421.2012.00411.x PMID: 22309616

21. Imamura T, Suzuki A, Lupisan S, Okamoto M, Aniceto R, Egos RJ, et al. Molecular evolution of enterovirus 68 detected in the Philippines. PLoS One. 2013;8(9):e74221. DOI: 10.1371/journal. pone.0074221 PMID: 24073203

22. Meijer A, van der Sanden S, Snijders BE, Jaramillo-Gutierrez $\mathrm{G}$, Bont L, van der Ent CK, et al. Emergence and epidemic occurrence of enterovirus 68 respiratory infections in The Netherlands in 2010. Virology. 2012;423(1):49-57. DOI: 10.1016/j.virol.2011.11.021 PMID: 22177700

23. Piralla A, Lilleri D, Sarasini A, Marchi A, Zecca M, Stronati M, et al. Human rhinovirus and human respiratory enterovirus (EV68 and EV104) infections in hospitalized patients in Italy, 2008-2009. Diagn Microbiol Infect Dis. 2012;73(2):162-7. DOI: 10.1016/j.diagmicrobio.2012.02.019 PMID: 22494556

24. Tokarz R, Kapoor V, Wu W, Lurio J, Jain K, Mostashari F, et al. Longitudinal molecular microbial analysis of influenza-like illness in New York City, May 2009 through May 2010. Virol J. 2011;8(1):288. DOI: 10.1186/1743-422X-8-288 PMID: 21658237

25. Renois F, Bouin A, Andreoletti L. Enterovirus 68 in pediatric patients hospitalized for acute airway diseases.J Clin Microbiol. 2013;51(2):640-3. DOI: 10.1128/JCM.02640-12 PMID: 23224095

26. Drews SJ, Simmonds K, Usman HR, Yee K, Fathima S, Tipples G, et al. Characterization of enterovirus activity, including that of enterovirus D68, in pediatric patients in Alberta, Canada, in 2014. J Clin Microbiol. 2015;53(3):1042-5. DOI: 10.1128/ JCM.02982-14 PMID: 25588657
27. Reiche J, Böttcher S, Diedrich S, Buchholz U, Buda S, Haas W, et al. Low-level Circulation of Enterovirus D68-Associated Acute Respiratory Infections, Germany, 2014. Emerg Infect Dis. 2015;21(5):837-41. DOI: 10.3201/eid2105.141900 PMID: 25898320

28. Messacar K, Schreiner TL, Maloney JA, Wallace A, Ludke J, Oberste MS, et al. A cluster of acute flaccid paralysis and cranial nerve dysfunction temporally associated with an outbreak of enterovirus D68 in children in Colorado, USA. Lancet. 2015;385(9978):1662-71. DOI: 10.1016/S01406736(14)62457-0 PMID: 25638662

29. Greninger AL, Naccache SN, Messacar K, Clayton A, Yu G, Somasekar S, et al. A novel outbreak enterovirus D68 strain associated with acute flaccid myelitis cases in the USA (2012-14): a retrospective cohort study. Lancet Infect Dis. 2015;15(6):671-82. DOI: 10.1016/S1473-3099(15)70093-9 PMID: 25837569

30. Division of Viral Diseases, National Centers for Immunization and Respiratory Diseases, CDC; Division of Vector-Borne Diseases, Division of High-Consequence Pathogens and Pathology, National Center for Emerging and Zoonotic Infectious Diseases, CDC; Children's Hospital Colorado; Council of State and Territorial Epidemiologists. Notes from the field: acute flaccid myelitis among persons aged $\leq 21$ years - United States, August 1-November 13, 2014.MMWR Morb Mortal Wkly Rep. 2015;63(53):1243-4.PMID: 25577990

31. Respiratorisches Viren - Netzwerk (RespVir). German [Accessed 20 May 2015]. Available from: http://EVsurv.rki.de

32. Blomqvist S, Savolainen C, Råman L, Roivainen M, Hovi T. Human rhinovirus 87 and enterovirus 68 represent a unique serotype with rhinovirus and enterovirus features.J Clin Microbiol. 2002;40(11):4218-23. DOI: $10.1128 / J C M .40 .11 .4218$ 4223.2002 PMID: 12409401

33. Oberste MS, Maher K, Schnurr D, Flemister MR, Lovchik $\mathrm{JC}$, Peters $\mathrm{H}$, et al. Enterovirus 68 is associated with respiratory illness and shares biological features with both the enteroviruses and the rhinoviruses. J Gen Virol. 2004;85(Pt 9):2577-84. DOI: 10.1099/vir.0.79925-0 PMID: 15302951

34. Imamura T, Okamoto M, Nakakita S, Suzuki A, Saito M, Tamaki R, et al. Antigenic and receptor binding properties of enterovirus 68. J Virol. 2014;88(5):2374-84. DOI: 10.1128 / JVI.03070-13 PMID: 24371050

\section{License and copyright}

This is an open-access article distributed under the terms of the Creative Commons Attribution (CC BY 4.0) Licence. You may share and adapt the material, but must give appropriate credit to the source, provide a link to the licence, and indicate if changes were made.

This article is copyright of the authors, 2016. 